\title{
Chemical Evolution of Strongly Interacting Quark-Gluon Plasma
}

\author{
Ying-Hua Pan $^{1}$ and Wei-Ning Zhang ${ }^{1,2}$ \\ ${ }^{1}$ Department of Physics, Harbin Institute of Technology, Harbin, Heilongjiang 150006, China \\ ${ }^{2}$ School of Physics and Optoelectronic Technology, Dalian University of Technology, Dalian, Liaoning 116024, China \\ Correspondence should be addressed to Ying-Hua Pan; panyh@hit.edu.cn
}

Received 23 August 2014; Accepted 12 November 2014; Published 24 November 2014

Academic Editor: Sally Seidel

Copyright (c) 2014 Y.-H. Pan and W.-N. Zhang. This is an open access article distributed under the Creative Commons Attribution License, which permits unrestricted use, distribution, and reproduction in any medium, provided the original work is properly cited. The publication of this article was funded by SCOAP ${ }^{3}$.

\begin{abstract}
At very initial stage of relativistic heavy ion collisions a wave of quark-gluon matter is produced from the break-up of the strong color electric field and then thermalizes at a short time scale $(\sim 1 \mathrm{fm} / \mathrm{c})$. However, the quark-gluon plasma (QGP) system is far out of chemical equilibrium, especially for the heavy quarks which are supposed to reach chemical equilibrium much late. In this paper a continuing quark production picture for strongly interacting QGP system is derived, using the quark number susceptibilities and the equation of state; both of them are from the results calculated by the Wuppertal-Budapest lattice QCD collaboration. We find that the densities of light quarks increase by $75 \%$ from the temperature $T=400 \mathrm{MeV}$ to $T=150 \mathrm{MeV}$, while the density of strange quark annihilates by $18 \%$ in the temperature region. We also offer a discussion on how this late production of quarks affects the final charge-charge correlations.
\end{abstract}

\section{Introduction}

The ultrarelativistic heavy-ion collisions at the Relativistic Heavy Ion Collider (RHIC) and the Large Hadron Collider (LHC) provide a unique opportunity for scientists to investigate the properties of the quark-gluon plasma (QGP) in laboratory, which is believed to be a form of early universe after the big bang. In these collisions, the heavy nuclei can be depicted in the center-of-mass frame (CMF) of system as two Lorenz contracted pancakes. Most of the baryons are expected to be carried away by the receding pancakes, while in the region of the collisions large energy is deposited in the form of quarks and gluons $[1,2]$. A large amount of quark-antiquark pairs will be produced from the breakup of the strong color electric field established between two receding pancakes, when the energy in the field is larger than the pair production threshold [3-5]. The collisions between the newborn constituents are violent enough to drive initial collision products to a new deconfined quark-gluon plasma at a short time scale, $\tau_{0}(\sim 1 \mathrm{fm} / \mathrm{c})$ [6]. The initial QGP is gluon rich and thermalizes very rapidly. But complete chemical equilibrium will take much longer time. For quarks, the rate of chemical equilibrium is much lower than that of gluon, because the relevant QCD cross sections are 2-3 times smaller $[7,8]$. Another process of late production of quarks is the strange-quark production, which will take place up to 5$10 \mathrm{fm} / \mathrm{c}$ when the local temperature drops to near $160 \mathrm{MeV}$ [9].

The quark late stage production can place a significant impact on the correlations between charges and be investigated through the balance functions [10]. The late production of quarks causes the widths of balance functions smaller, because the later the charges produce, the closer the collective flow will constrain charges in momentum space. In [11], Pratt extends the theory of balance function to the scenario involving two-wave production of quarks. The first wave takes place in the first $\mathrm{fm} / \mathrm{c}$ of the collisions when gluons thermalize into quark-gluon plasma (QGP), and the second wave ensues at hadronization, $5-10 \mathrm{fm} / \mathrm{c}$, after a roughly isentropic expansion that roughly conserves the number of quarks [11]. However this canonical picture of the evolution of the QGP in high-energy heavy-ion collisions is still oversimplified. In principle, the number of quarkantiquark pairs could adjust continually up to the time when 
full chemical equilibrium reaches. This time may occur late; for instance, for strangeness pairs, the saturation of number happens at 5-10 fm/c after collision [9].

The experimental results available so far show that the hot QCD matter exhibits robust flow phenomena, which are well and consistently described by near-ideal relativistic hydrodynamics $[1,12]$. This arouses the suspicion of applicability of perturbative QCD to the hot and dense matter at the region of temperature $T_{c}<T<3 T_{c}$. To account for nonperturbative effect, lattice QCD provides a reliable method. Numerical simulations of QCD thermodynamics on the lattice are reaching unprecedented level of accuracy. The Wuppertal-Budapest lattice QCD collaboration computed the QCD equation of state with $2+1$ staggered flavor [13]. Their results of the large trace anomaly hints at a strongly interacting system instead of the weakly interacting parton gas system. For the strongly interacting system, it is a big challenge to calculate the number densities of quarks directly. However, lattice QCD provide a technology to calculate the quark number susceptibilities with high accuracy [14]. In the classical weakly interacting gas limit, the diagonal quadratic susceptibilities are related to the number densities of quarks. This provides a possibility to infer the chemical composition of quark-gluon matter at strongly interacting region. Although the interaction between constituents in the quark-gluon system at temperature $T_{c}<T<3 T_{c}$ is not weak enough, the hard thermal loop (HTL) perturbation theory motivates and justifies a picture of weakly interacting quasiparticle at this region of temperature. In quasiparticle model, the interaction between constituents is encoded in the quasiparticle masses and the quasiparticles behave like a free gas of massive constituents. Plumari et al. further obtained that quasiparticle model can reproduce the susceptibilities by using the quark and gluon masses that come from the HTL formalism [15].

In this paper, we try to use the susceptibilities calculated from lattice QCD to investigate the evolution of the number densities of quarks. For the expansion of quarkgluon matter, the combination of boost invariant longitudinal expansion and cylindrical symmetric transverse expansion is considered. The equation of state (EOS) from lattice QCD results [13] is used in our hydrodynamic calculations. The rest of this paper is organized as follows. In Section 2, we will describe the calculation method used in this work. In Section 3, we will present our results for a continuing, late quark production picture and make discussions. Finally, we will give the summary and conclusions in Section 4 .

\section{Calculation Method}

A system in the thermal equilibrium (for grand-canonical ensemble) is characterized by its partitions function,

$$
Z=\operatorname{Tr}\left[\exp \left(-\frac{H-\sum_{i} \mu_{i} N_{i}}{T}\right)\right],
$$

where $H$ is the Hamiltonian of the system and $N_{i}$ and $\mu_{i}$ are the conserved charges and the chemical potentials, respectively. In case of three flavor quarks, these are strangeness, baryon number, and electric charge or, equivalently, the three quark flavors: up, down, and strange. In this work, we focus on the quark number susceptibilities. So $N_{i}$ denotes the (net) number of quarks and $\mu_{i}$ is the corresponding chemical potential. The mean and the covariance are then expressed in terms of derivatives of the partition function with respect to the appropriate chemical potentials,

$$
\begin{gathered}
\left\langle N_{i}\right\rangle=T \frac{\partial}{\partial \mu_{i}} \log (Z), \\
\left\langle\delta N_{i} \delta N_{j}\right\rangle=T^{2} \frac{\partial^{2}}{\partial \mu_{i} \partial \mu_{j}} \log (Z) \equiv V T^{3} \chi_{i, j}^{2},
\end{gathered}
$$

where $\delta N_{i}=N_{i}-\left\langle N_{i}\right\rangle$. The susceptibilities is introduced by

$$
\chi_{i j}^{2}=\frac{1}{V T} \frac{\partial^{2}}{\partial \mu_{i} \partial \mu_{j}} \log (Z) .
$$

The diagonal susceptibilities, $\chi_{i, i}^{2}$, are a measure of the fluctuation of the system, whereas the off-diagonal susceptibilities, $\chi_{i, j}^{2}$, characterize the correlation between the conserved charges $N_{i}$ and $N_{j}$.

We assume the quark-gluon matter can be described by the weakly interacting quark-gluon quasiparticle gas and further neglect small correlations due to quantum statistics which are of the order of one percent for the temperatures under consideration $[16,17]$. Thus, the fluctuation of $N_{i}$, the (net) number of quarks, is given by

$$
\begin{aligned}
\left\langle\delta N_{i}^{2}\right\rangle & =\left\langle\left(\delta N_{q}-\delta N_{\bar{q}}\right)^{2}\right\rangle \\
& =\left[\left\langle\delta N_{q}^{2}\right\rangle+\left\langle\delta N_{\bar{q}}^{2}\right\rangle\right] \\
& =\left[\left\langle N_{q}\right\rangle+\left\langle N_{\bar{q}}\right\rangle\right]=\widehat{N}_{i} .
\end{aligned}
$$

Since $\left\langle\delta N_{q} \delta N_{\bar{q}}\right\rangle=0$ and $\left\langle\delta N^{2}\right\rangle=\langle N\rangle$ for an ideal gas, $\widehat{N}_{i}$ is the sum of the number of quarks and antiquarks for flavor $i$. In case of flavor $n_{f}=2+1, i$ can be $u(d)$ for the light quark and $s$ for the strange quark. By substituting (4) to (2), we can relate $\widehat{N}_{i}$ to the corresponding diagonal susceptibilities defined by (3):

$$
\widehat{N}_{i}=\left\langle\delta N_{i}^{2}\right\rangle=T^{2} \frac{\partial^{2}}{\partial \mu_{i}^{2}} \log (Z)=V T^{3} \chi_{i, i}^{2}
$$

As mentioned above, the susceptibilities, $\chi_{i, j}^{2}$, are investigated a lot in lattice QCD simulation. In [14], Borsányi et al. computed the $\chi_{i, j}^{2}$ at the region of temperature $125-400 \mathrm{MeV}$ for flavor $n_{f}=2+1$ with the physical quark mass and performed the continuum extrapolation to remove artificial cut-off effect. Their results are presented in Figure 1.

To derive the evolution of chemical composition, we need to overcome the problem with how to describe the expansion of quark-gluon system. In the heavy ion collisions at the RHIC and the LHC, the amount of net charges is negligibly small in the central rapidity of the collisions. For simplicity, we use the hydrodynamics with zero net-baryon densities 


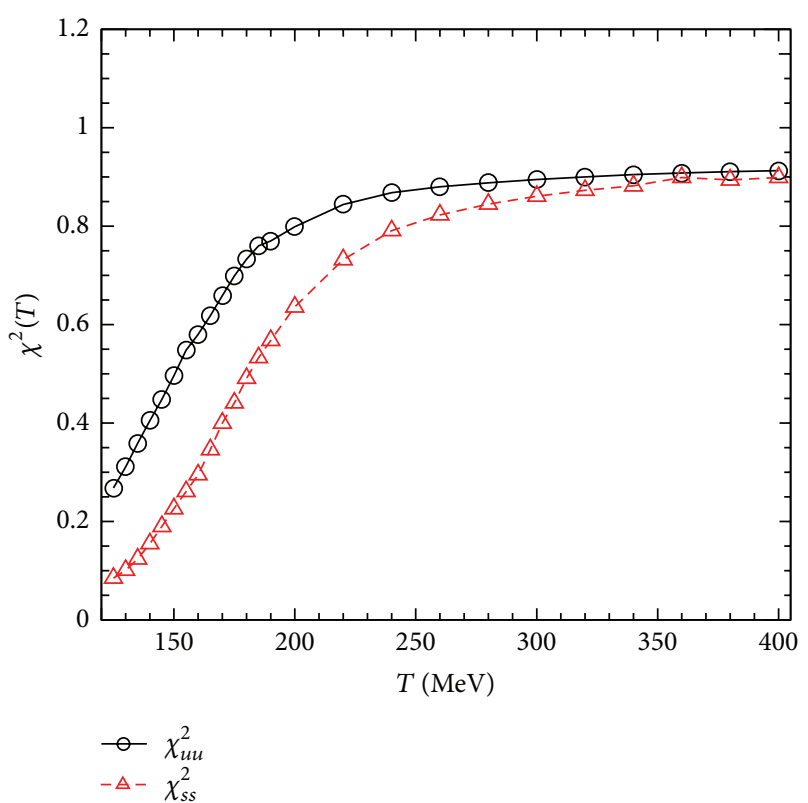

FIGURE 1: The continuum limit of light and strange quark susceptibilities from [14] (color online).

to describe the system expansion, which means neglecting the effect of those small net charges on the temperature and velocity fields of hydrodynamics. We assume the longitudinal boost invariant expansion and combine with cylindrically symmetric expansion in transverse direction, which is the Bjorken picture [18] in ultrarelativistic heavy-ion collisions. In this scheme, we can have two related equations written in cylindrical coordinates which govern the expansion of a neutral system (free of net charge) of QGP:

$$
\begin{gathered}
v_{r} \frac{\partial \ln (T)}{\partial t}+\frac{\partial \ln (T)}{\partial r}+\frac{1}{1-v_{r}^{2}}\left(\frac{\partial v_{r}}{\partial t}+v_{r} \frac{\partial v_{r}}{\partial r}\right)=0, \\
\frac{\partial \ln (s)}{\partial t}+v_{r} \frac{\partial \ln (s)}{\partial r}+\frac{1}{1-v_{r}^{2}}\left(v_{r} \frac{\partial v_{r}}{\partial t}+\frac{\partial v_{r}}{\partial r}\right)+\frac{v_{r}}{r}+\frac{1}{t}=0,
\end{gathered}
$$

where we have two independent variables, $r$ and $t$, and three dependent unknown quantities, temperature $T(r, t)$, entropy density $s(r, t)$, and transverse flow velocity $v_{r}(r, t)$. To solve these three unknown quantities, we need to supplement EOS.

We implement the EOS in [13], where the related quantities are computed with the same physics setup as ones which are used to calculate the susceptibilities [14]. In [13] a parametrization of the trace anomaly as a function of the temperature is given by

$$
\frac{I(T)}{T^{4}}=\exp \left[-\frac{h_{1}}{\widetilde{T}}-\frac{h_{2}}{\widetilde{T}^{2}}\right]\left(h_{0}+\frac{f_{0}\left[\tanh \left(f_{1} \widetilde{T}+f_{2}\right)+1\right]}{1+g_{1} \widetilde{T}+g_{2} \widetilde{T}^{2}}\right),
$$

where dimensionless $\widetilde{T}$ variable is defined as $\widetilde{T}=T / 200 \mathrm{MeV}$. The parameters are listed in Table 1 . The consistency between the calculated results and the parametrization is shown in

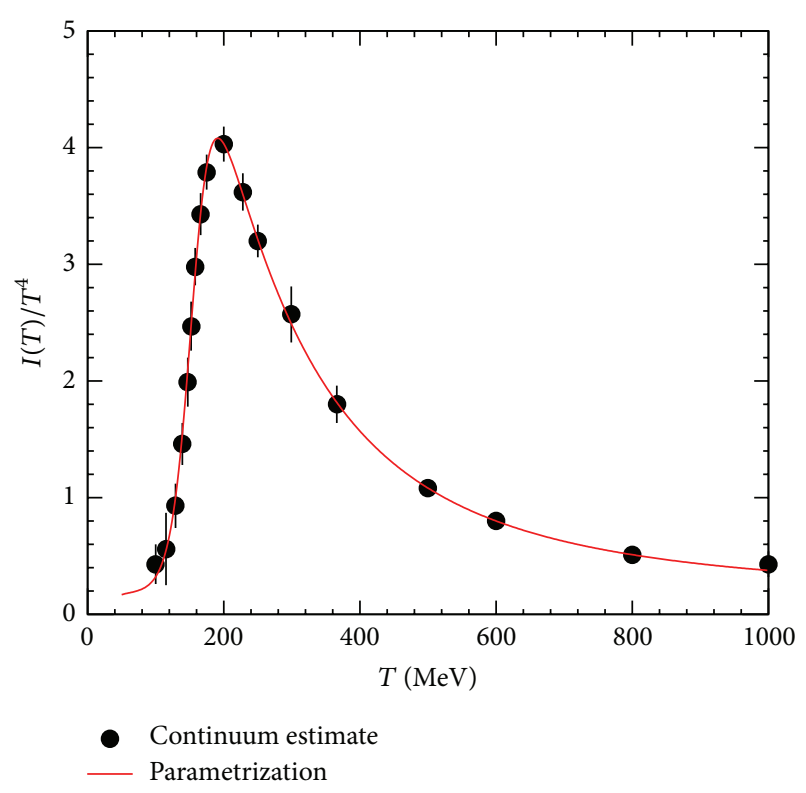

FIgURE 2: The trace anomaly normalized by $T^{4}$ with the parametrization of (7) using the parameters in Table 1 for $n_{f}=2+1$ [13] (color online).

TABLE 1: Parameters of the function in (7) describing the trace anomaly for $n_{f}=2+1[13]$.

\begin{tabular}{cccccccc}
\hline$h_{0}$ & $h_{1}$ & $h_{2}$ & $f_{0}$ & $f_{1}$ & $f_{2}$ & $g_{1}$ & $g_{2}$ \\
\hline 0.1396 & -0.1800 & 0.0350 & 2.76 & 6.79 & -5.29 & -0.47 & 1.04 \\
\hline
\end{tabular}

Figure 2. From this parametrization the normalized pressure can be obtained by the definite integral:

$$
\frac{P(T)}{T^{4}}=\int_{0}^{T} \frac{d T^{\prime}}{T^{\prime}} \cdot \frac{I\left(T^{\prime}\right)}{T^{\prime 4}} .
$$

With the help of the definition of the trace anomaly, $I=$ $\varepsilon-3 P$, we can have energy density, $\epsilon$, and the squared velocity of sound, $C_{s}^{2}$ :

$$
\begin{gathered}
\epsilon=I+3 P, \\
C_{s}^{2}(T)=\frac{\partial P}{\partial \varepsilon}=\frac{1}{(\partial I / \partial P)+3} .
\end{gathered}
$$

The numerical results of the squared velocity of sound and the pressure are presented in Figures 3(a) and 3(b).

In Figures 4(a) and 4(b) we present the hydrodynamic solution of the transverse expansion (at $z=0$ ) for the QGP system with Bjorken cylinder. In the calculation, we assume the initial time $\tau_{0}=0.1 r_{0}$ and take the following initial temperature and velocity profiles as in [19]:

$$
\begin{array}{cl}
T(r, t)=T_{0}, & r<r_{0}, \\
T(r, t)=0, & r>r_{0}, \\
v(r, t)=0, & r<r_{0}, \\
v(r, t)=1, & r>r_{0},
\end{array}
$$




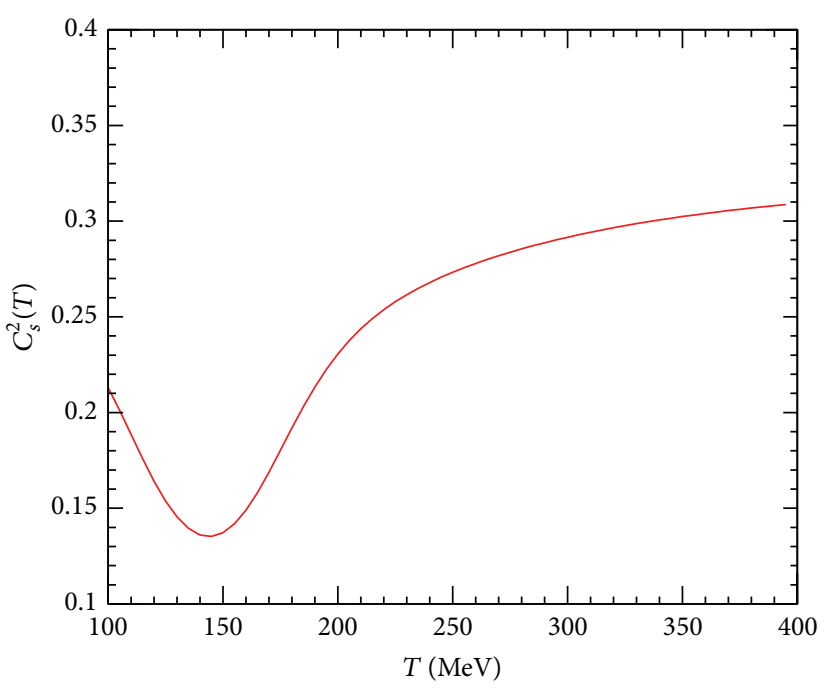

(a)

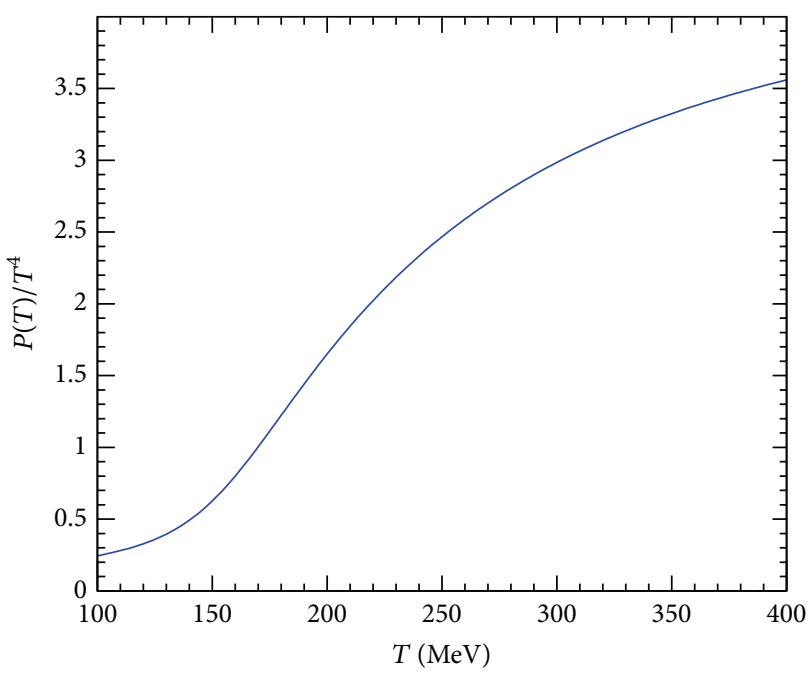

(b)

FIgURE 3: (a) The squared velocity of sound varies as a function of temperature $T$ and (b) the pressure varies as a function of temperature $T$ (color online).

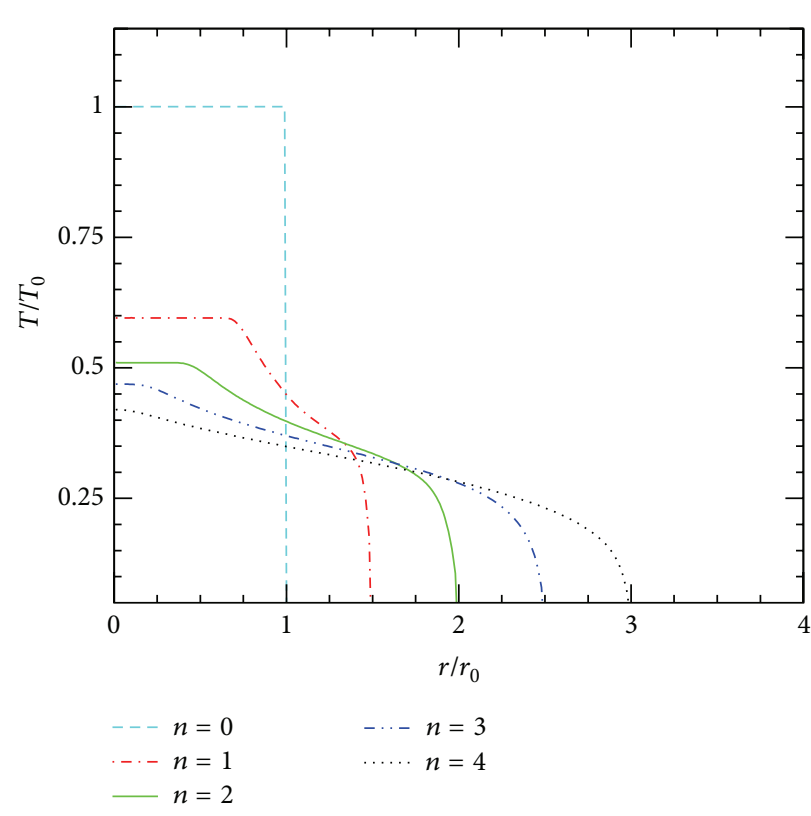

(a)

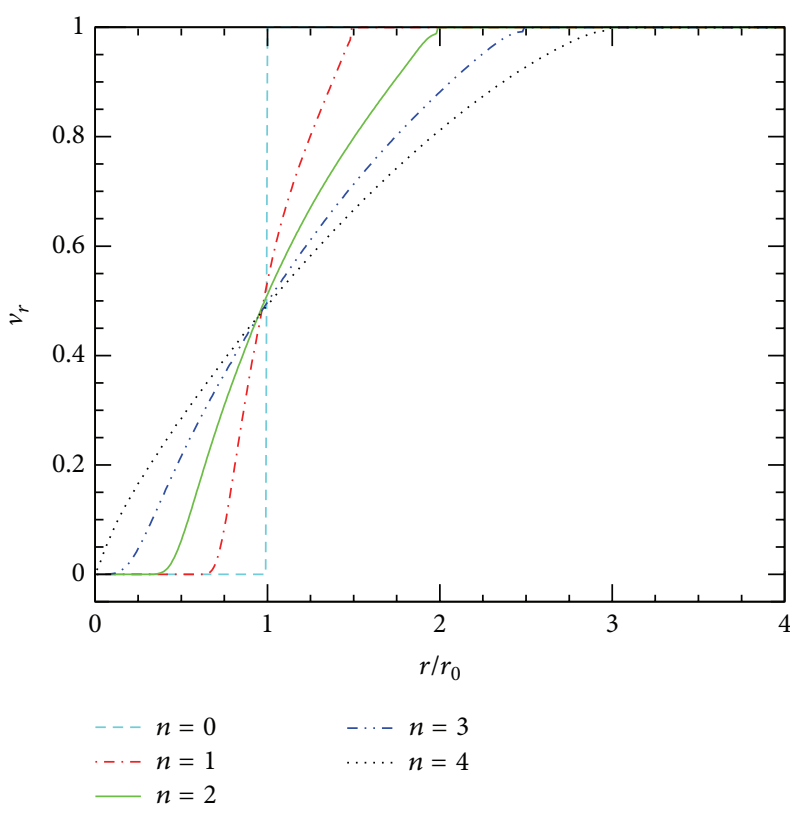

(b)

FIGURE 4: The profiles of temperature (a) and transverse velocity (b) for the expanding QGP system with Bjorken cylinder at different time $\left(t_{n}=\tau_{0}+0.5 n \lambda r_{0}, \lambda=0.99\right)$ (color online).

where $T_{0}$ and $r_{0}$ are the initial temperature and transverse radius of the system. They are taken to be $400 \mathrm{MeV}$ and $7 \mathrm{fm}$ in our calculations, respectively.

Once the evolution the quark-gluon system is available, we can derive the evolution of the rapidity densities of quark numbers, $n_{i}(\tau)=2 \pi \int r d r \widehat{N}_{i}(r, \tau) /\left[\pi r_{0}^{2} d \eta\right]$. For the Bjorken cylinder, we have from (5)

$$
n_{i}(\tau)=\frac{2}{r_{0}^{2}} \int r d r \tau T^{3}(r, \tau) \chi_{i, i}^{2}[T(r, \tau)] \gamma(r, \tau),
$$

where $\gamma\left(r^{\prime}, \tau\right)$ is Lorenz factor $1 / \sqrt{1-v_{r}^{2}}$ for transverse flow. In this paper, we only care about the chemical evolution of QGP, so the integral range of $r$ is determined by $T(r, \tau)>T_{c}$ for each time. Here $T_{c}$ is selected to be $150 \mathrm{MeV}$ as in [13].

\section{Results}

We first show in Figure 5 the rapidity densities of quark numbers, $n_{i}(\tau)$, for light and strange quarks. The time dependence 


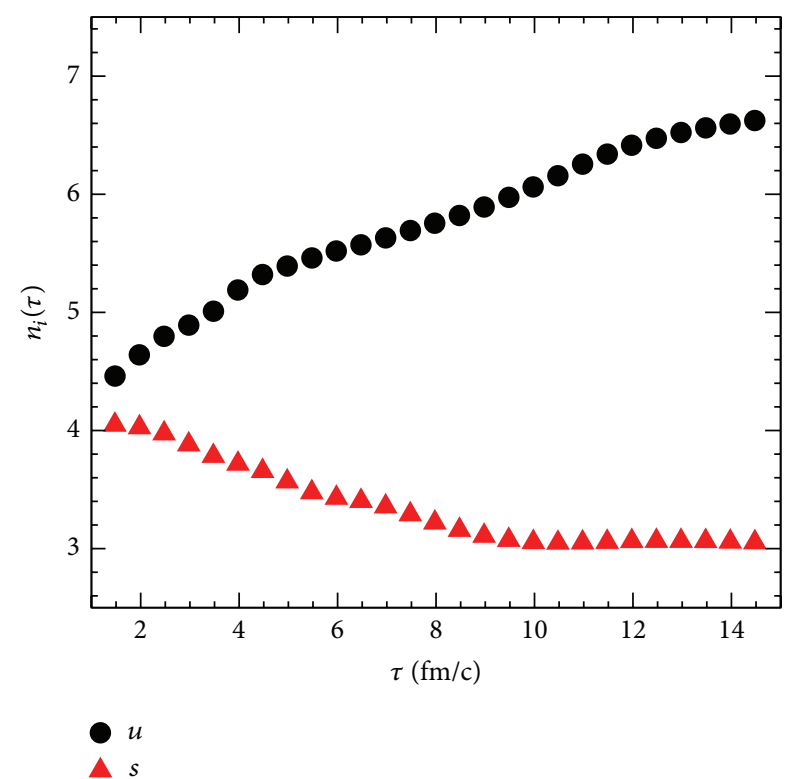

FIGURE 5: The time dependence of the rapidity densities of quark numbers, $n_{i}(t)$, defined in (11) (color online).

of the number density for the light quark deviates from that for the strange quark at very early stage, when $\chi_{u u}^{2}$ starts to deviate from $\chi_{s s}^{2}$ slightly. $n_{u}(\tau)$ arises with time rapidly from $\tau_{0}$ to $14.6 \mathrm{fm} / \mathrm{c}$ by $75 \%$, while $n_{s}(\tau)$ drops by $18 \%$ at that time after a rise during the first $1.5 \mathrm{fm} / \mathrm{c}$. This observable is cut-off at $\tau=14.6 \mathrm{fm} / \mathrm{c}$, because at the time the temperature reaches the critical temperature $T_{c}=150 \mathrm{MeV}$ defined by WuppertalBudapest group [13].

The ratio of number of strange quark to the number of light quark is an important indication of chemical composition, which can be calculated using the measured ratio, $K / \pi$ in the experiments:

$$
R_{s / u}=\frac{n_{s}}{n_{u}+n_{d}} \approx \frac{R_{K / \pi}}{\left(1.5+R_{K / \pi}\right)} .
$$

The approximation is valid for the system which most comprises of $K, \pi$ and has the same amount of antiparticles as particles. Our results on $R_{s / u}$ are shown in Figure 6. The value of $R_{s / u}$ is 0.22 at $T_{c}=150 \mathrm{MeV}$ when the quark-gluon system terminates in our calculation. This value is about twice bigger than the values 0.09 and 0.1 , calculated from the measured $K / \pi$ in the experiments at the LHC [2] and at the RHIC [20], respectively. This indicates that the chemical composition still alters slightly during the hadron gas through strange particle annihilation, decay, and baryon annihilation $[21,22]$.

The late production mechanism of quarks can play a significant impact on the charge-charge correlation of QGP. The motion of the balancing pair (quark and antiquark) which are produced at the same point of space-time coordinate can be separated into a collective mode, due to the collective expansion of the system, and a diffusive mode, due to the collisions with the surrounding medium. One can study the total motion of quarks by overriding the diffusive motion on a collective flow $u^{\mu}$, which can be determined by hydrodynamic

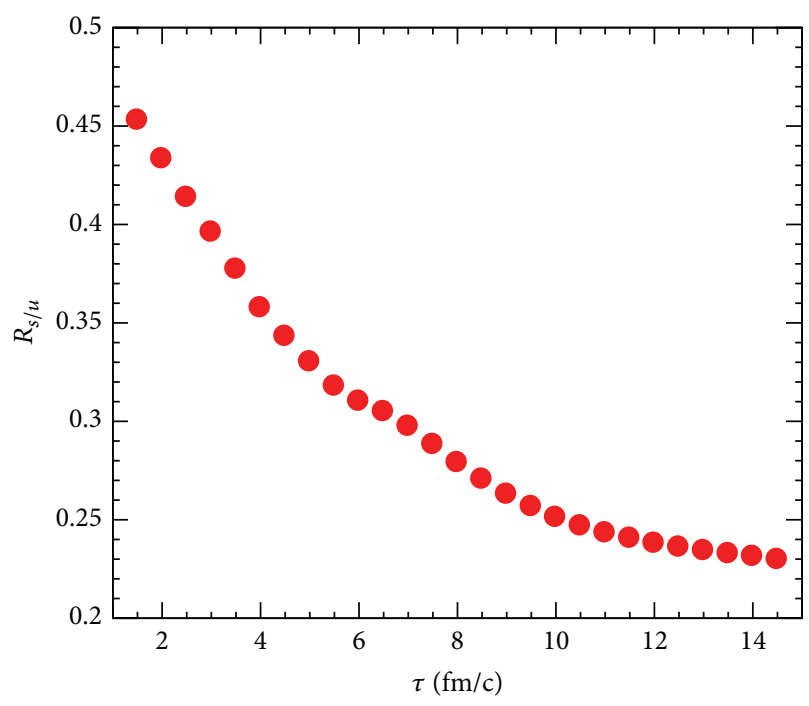

Figure 6: The time dependence of the ratio of the number of strange quarks to the number of light quarks (color online).

calculations. In [23], by overriding the diffusive motion on a longitudinal Bjorken flow, the following equation of the charge-charge correlation, $g_{a b}$, is derived:

$$
\left(\tau_{d} \frac{\partial^{2}}{\partial \tau^{2}}+\frac{\partial}{\partial \tau}-\frac{D}{\tau^{2}} \frac{\partial^{2}}{\partial \eta_{a}^{2}}-\frac{D}{\tau^{2}} \frac{\partial^{2}}{\partial \eta_{r}^{2}}\right) g_{a b}=0 .
$$

Here $\tau_{d}$ is the relaxation time for diffusion, $\eta_{r}=\eta_{1}-\eta_{2}$ is the relative rapidity, $\eta_{a}=\left(\eta_{1}+\eta_{2}\right) / 2$ is the average rapidity of the two particles, $D=\tau / 3 \alpha$, and $\alpha$ is a parameter of local scattering rate. One can find $\sigma$, the rapidity width of $g_{a b}$ [23]:

$$
\sigma^{2}=\left\langle\left(\eta_{r}-\left\langle\eta_{r}\right\rangle\right)^{2}\right\rangle=2 \Delta V\left(\tau^{\prime}, \tau^{\prime \prime}\right),
$$

by multiplying (13) by $\eta_{r}^{2}$ and integrating over both variables. Here $\Delta V$ is given by

$$
\Delta V\left(\tau^{\prime}, \tau^{\prime \prime}\right)=\frac{2}{3(\alpha-1)}\left\{\log \frac{\tau^{\prime \prime}}{\tau^{\prime}}-\frac{1}{\alpha-1}\left[1-\left(\frac{\tau^{\prime}}{\tau^{\prime \prime}}\right)^{\alpha-1}\right]\right\} .
$$

The width $\sigma$ is determined by the time $\tau^{\prime}$ at which charges are created, the time $\tau^{\prime \prime}$ at which the diffusion of charges freezes, and the local scattering rate $\alpha$.

The local scattering rate characterizes the ability of restoring local equilibrium for a source cell. Generally a system created by a nuclei-nuclei collision with a higher CMF energy has a larger $\alpha$ because it has a larger local densities and a higher temperature. The samples of $\sigma^{2}$ for three selected $\alpha$ are shown as functions of $\tau^{\prime}$ in Figure 7. The charges created at a later time diffuse with a smaller width $\sigma$. One of the reasons for that is that the later the charges are created, the more they are constrained by the collective flow. On the other hand, the diffusion time for the charges created later is smaller. For a fixed $\tau^{\prime}$, the value of $\sigma$ for a larger $\alpha$ is smaller. This is because the balancing pair in a source cell with a larger local scattering rate is harder to diffuse. 


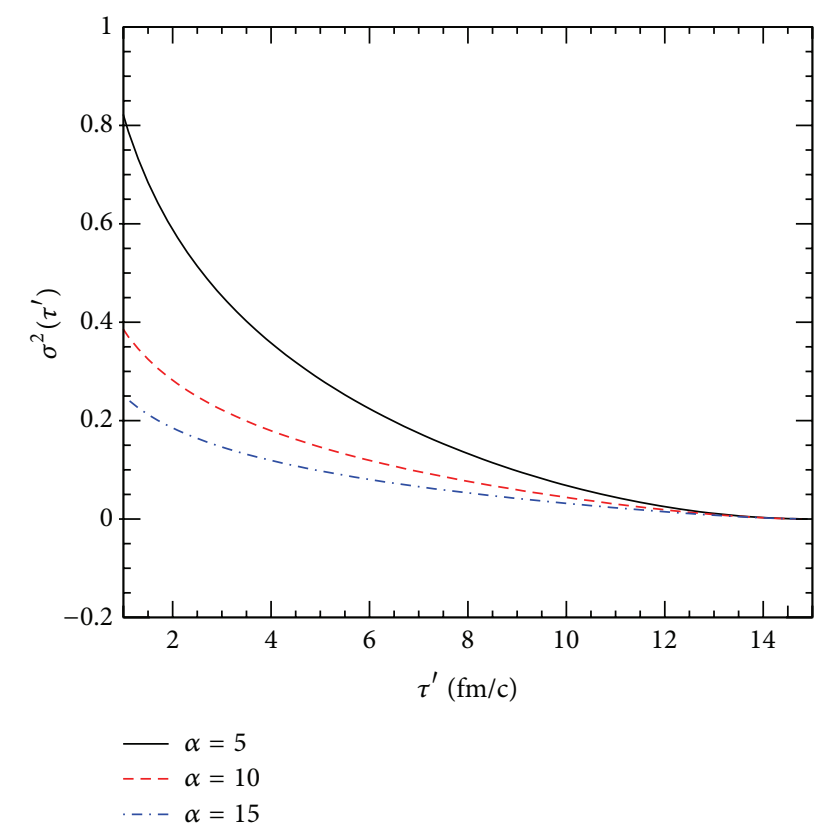

FIGURE 7: The width $\sigma$ varies as a function of $\tau^{\prime}$, the time at which charges are created, and three curves correspond to three different local scattering rate $\alpha$ (color online).

One can expect that $g_{a b}^{\mathrm{QGP}}$ has the following integral formulism with respect to time because of continuous creation or annihilation of charges:

$$
g_{a b}^{\mathrm{QGP}}(\Delta \eta, \tau)=-\int_{\tau_{0}}^{\tau} d \tau^{\prime} \frac{d n_{a b}\left(\tau^{\prime}\right)}{d \tau^{\prime}} f\left(\Delta \eta, \tau^{\prime}, \tau\right)
$$

where $f\left(\Delta \eta, \tau^{\prime}, \tau\right)$ describes the normalized probability of charges diffusing away from one another by a distance $\Delta \eta$ at time $\tau$ given they were created at $\tau^{\prime}$. Generally the $f$ can be determined by solving the diffusion equation. Here we assume the diffusion of balancing charges has a Gaussian-like structure:

$$
f\left(\Delta \eta, \tau^{\prime}, \tau\right)=\frac{e^{-(\Delta \eta)^{2} / 2 \sigma_{\mathrm{QGP}}^{2}\left(\tau^{\prime}, \tau\right)}}{\left[2 \pi \sigma_{\mathrm{QGP}}^{2}\left(\tau^{\prime}, \tau\right)\right]^{1 / 2}},
$$

where $\sigma_{\mathrm{QGP}}^{2}$ is calculated by (14).

The rapidity width of $g_{a b}^{\mathrm{QGP}}$ is defined:

$$
\Delta \widetilde{\eta}=\sqrt{\left\langle(\Delta \eta)^{2}\right\rangle-\langle\Delta \eta\rangle^{2}}
$$

The systems with different local scattering rate $\alpha$ have different rapidity width of $g_{a b}^{\mathrm{QGP}}$. In Figure 8 , we plot the $\Delta \widetilde{\eta}$ of $g_{a b}^{\mathrm{QGP}}$ as a function of local scattering rate, $\alpha$. The symbols triangle and square are for the correlations of strange quarks and light quarks. The width of $g_{u u}^{\mathrm{QGP}}$ has a much larger dependence on the local scattering rate $\alpha$ than that of $g_{s s}^{\mathrm{QGP}}$. This is because

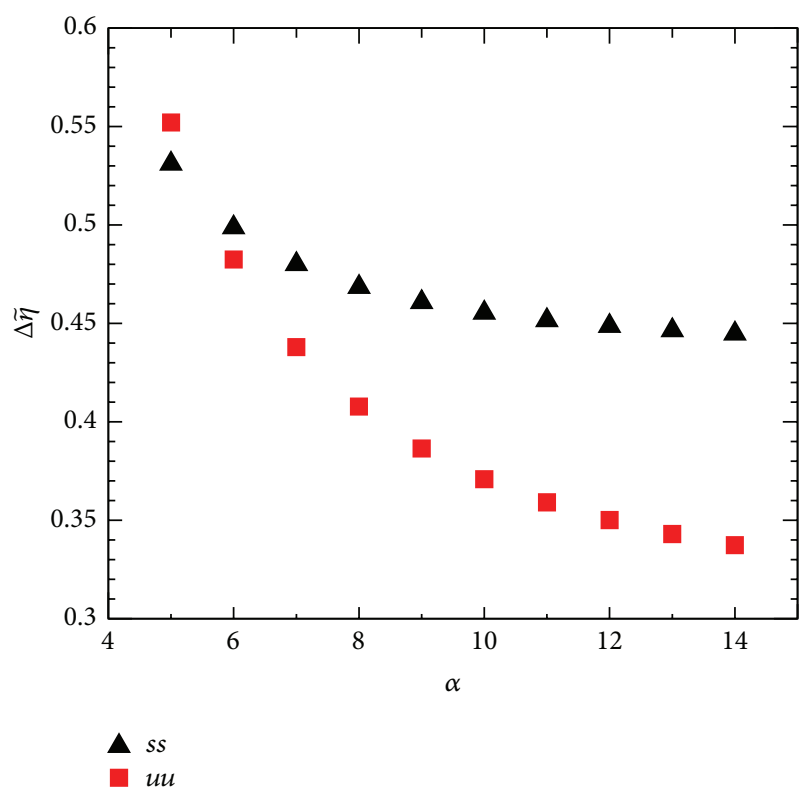

FIGURE 8: The width of $g_{a b}^{\mathrm{QGP}}, \Delta \widetilde{\eta}$, varies as a function of local scattering rate $\alpha$ (color online).

strange quark annihilates by $18 \%$ while light quark increases by $75 \%$ during the evolution of QGP.

The different dependences of $\Delta \widetilde{\eta}$ on $\alpha$ between strange quark and light quark are expected to appear in the correlations of final particles in experiments, if the correlations in QGP are not washed out by the processes afterwards. Because the correlations of $K^{+} K^{-}$carry on the correlation of strange quark, the balance functions of $K^{+} K^{-}$are supposed to have less dependence on the centrality than that of the balance functions of $\pi^{+} \pi^{-}$. This is consistent with the findings on balance functions in RHIC experiments [24].

\section{Summary}

In this paper we have calculated the evolution of chemical composition of the quark-gluon system with $2+1$ staggered quark flavors, using diagonal quark number susceptibilities and the EOS calculated by Wuppertal-Budapest group. Our results indicate that the number of the light quark increases by $75 \%$ while for strange quark it drops by $18 \%$ at the considered temperature region.

In our work a continuing charge production picture was used in the calculations. This continuing, late production mechanism will place a significant impact on conserved charge correlation. The correlations of strange particles have less dependence on centrality than that of hadrons comprised of light quarks. This can be tested by investigating the balance functions between particle species.

\section{Conflict of Interests}

The authors declare that there is no conflict of interests regarding the publication of this paper. 


\section{Acknowledgments}

Y.-H. Pan would like to thank Dr. S. Pratt for his kind hospitality and very useful discussion when visited at Michigan State University during the year 2011. This work was supported by the National Natural Science Foundation of China under Grant no. 11275037.

\section{References}

[1] J. Adams, M. M. Aggarwal, Z. Ahammed et al., "Experimental and theoretical challenges in the search for the quark-gluon plasma: The STAR Collaboration's critical assessment of the evidence from RHIC collisions," Nuclear Physics A, vol. 757, no. 1-2, pp. 102-183, 2005.

[2] B. Abelev, J. Adam, D. Adamova et al., "Centrality dependence of $\pi, K$, and $p$ production in $\mathrm{Pb}-\mathrm{Pb}$ collisions at $\sqrt{S_{N N}}=2.76$ TeV," Physical Review C, vol. 88, no. 4, Article ID 044910, 23 pages, 2013.

[3] J. W. Harris and B. Müller, "The search for the Quark-Gluon plasma," Annual Review of Nuclear and Particle Science, vol. 46, pp. 71-107, 1996.

[4] B. Blaizot, "Signals of the quark-gluon plasma in nucleusnucleus collisions," Nuclear Physics A, vol. 661, pp. 3-12, 1999.

[5] C. Y. Wong, Introduction to High-Energy Heavy Ion Collisions, World Scientific, Singapore, 1994.

[6] K. Adcox, S. S. Adler, S. Afanasiev et al., "Formation of dense partonic matter in relativistic nucleus-nucleus collisions at RHIC: experimental evaluation by the PHENIX Collaboration," Nuclear Physics A, vol. 757, pp. 184-283, 2005.

[7] T. S. Biró, E. Van Doorn, B. Müller, M. H. Thoma, and X.-N. Wang, "Parton equilibration in relativistic heavy ion collisions," Physical Review C, vol. 48, no. 3, pp. 1275-1284, 1993.

[8] K. Geiger and J. I. Kapusta, "Chemical equilibration of partons in high-energy heavy-ion collisions?” Physical Review D, vol. 47, no. 11, pp. 4905-4919, 1993.

[9] P. Koch, B. Müller, and J. Rafelski, "Strangeness in relativistic heavy ion collisions," Physics Reports, vol. 142, no. 4, pp. 167262, 1986.

[10] S. A. Bass, P. Danielewicz, and S. Pratt, "Clocking hadronization in relativistic heavy-ion collisions with balance functions," Physical Review Letters, vol. 85, no. 13, pp. 2689-2692, 2000.

[11] S. Pratt, "General charge balance functions: a tool for studying the chemical evolution of the quark-gluon plasma," Physical Review C, vol. 85, Article ID 014904, 2012.

[12] U. Heinz, "Early collective expansion: relativistic hydrodynamics and the transport properties of QCD matter," in Relativistic Heavy Ion Physics, vol. 23, pp. 240-292, Springer, Belin, Germany, 2010.

[13] S. Borsányi, G. Endrődi, Z. Fodor et al., "The QCD equation of state with dynamical quarks," Journal of High Energy Physics, vol. 2010, article 77, 2010.

[14] S. Borsányi, Z. Fodor, S. D. Katz, S. Krieg, C. Ratti, and K. Szabó, "Fluctuations of conserved charges at finite temperature from lattice QCD," Journal of High Energy Physics, vol. 2012, article 138, 2012.

[15] S. Plumari, W. M. Alberico, V. Greco, and C. Ratti, "Recent thermodynamic results from lattice QCD analyzed within a quasiparticle model," Physical Review D, vol. 84, no. 9, Article ID 094004, 10 pages, 2011.
[16] G. F. Bertsch, "Meson phase space density in heavy ion collisions from interferometry," Physical Review Letters, vol. 72, Article ID 2349, 1994.

[17] S. Jeon and V. Koch, "Event-by-event fluctuations," http://arxiv .org/abs/hep-ph/0304012.

[18] J. D. Bjorken, "Highly relativistic nucleus-nucleus collisions: the central rapidity region," Physical Review D, vol. 27, no. 1, pp.140151, 1983.

[19] D. H. Rischke and M. Gyulassy, "The time-delay signature of quark-gluon plasma formation in relativistic nuclear collisions," Nuclear Physics A, vol. 608, pp. 479-512, 1996.

[20] S. S. Adler, S. Afanasiev, C. Aidala et al., "Identified charged particle spectra and yields in Au+Aucollisions at $\sqrt{s_{N N}}=200$ GeV," Physical Review C, vol. 69, Article ID 034909, 2004.

[21] Y. H. Pan and S. Pratt, "Baryon annihilation and regeneration in heavy ion collisions," Physical Review C, vol. 89, Article ID 044911, 2014.

[22] Y. H. Pan and W. N. Zhang, "Resonance-like QGP signals displayed in general charge balance functions

", International Journal of Modern Physics E, vol. 23, no. 8, Article ID 1450036, 2014.

[23] M. A. Aziz and S. Gavin, "Causal diffusion and the survival of charge fluctuations in nuclear collisions," Physical Review C, vol. 70, Article ID 034905, 2004.

[24] M. M. Aggarwal, "Balance functions from $\mathrm{Au}+\mathrm{Au}, d+\mathrm{Au}$, and $p+p$ collisions at $\sqrt{s_{N N}}=200 \mathrm{GeV}$," Physical Review $C$, vol. 82, Article ID 024905, 2010. 

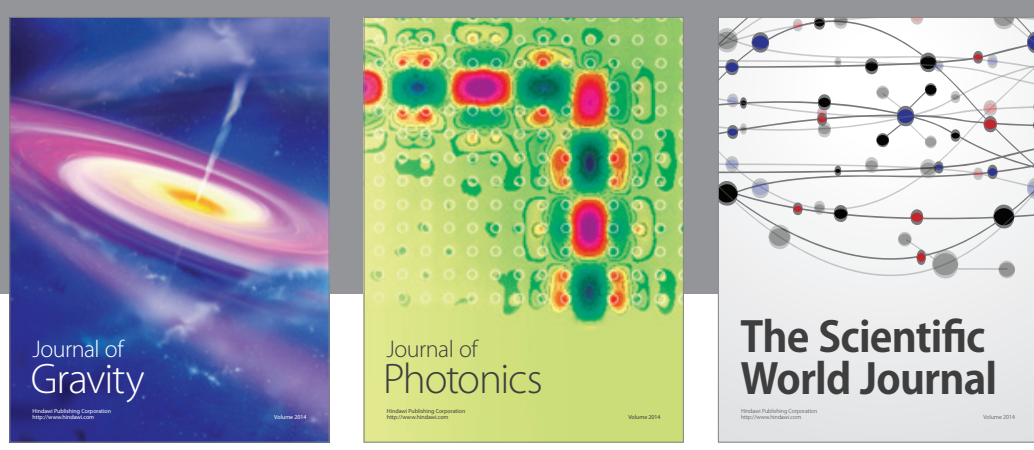

The Scientific World Journal
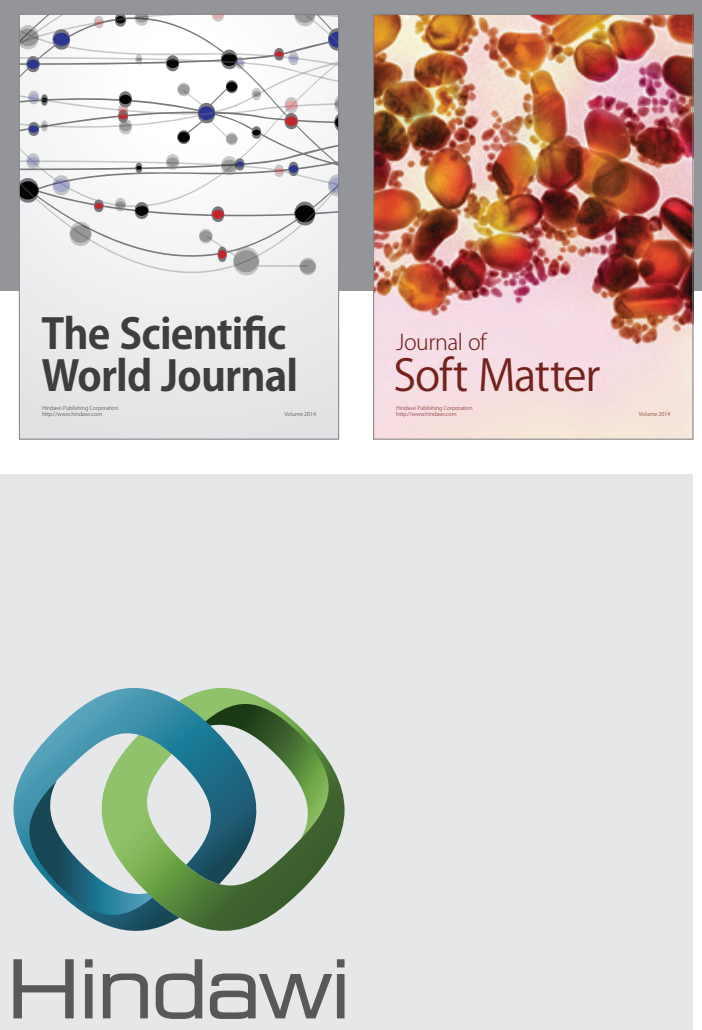

Submit your manuscripts at

http://www.hindawi.com

nternational Journal of

Statistical Mechanics
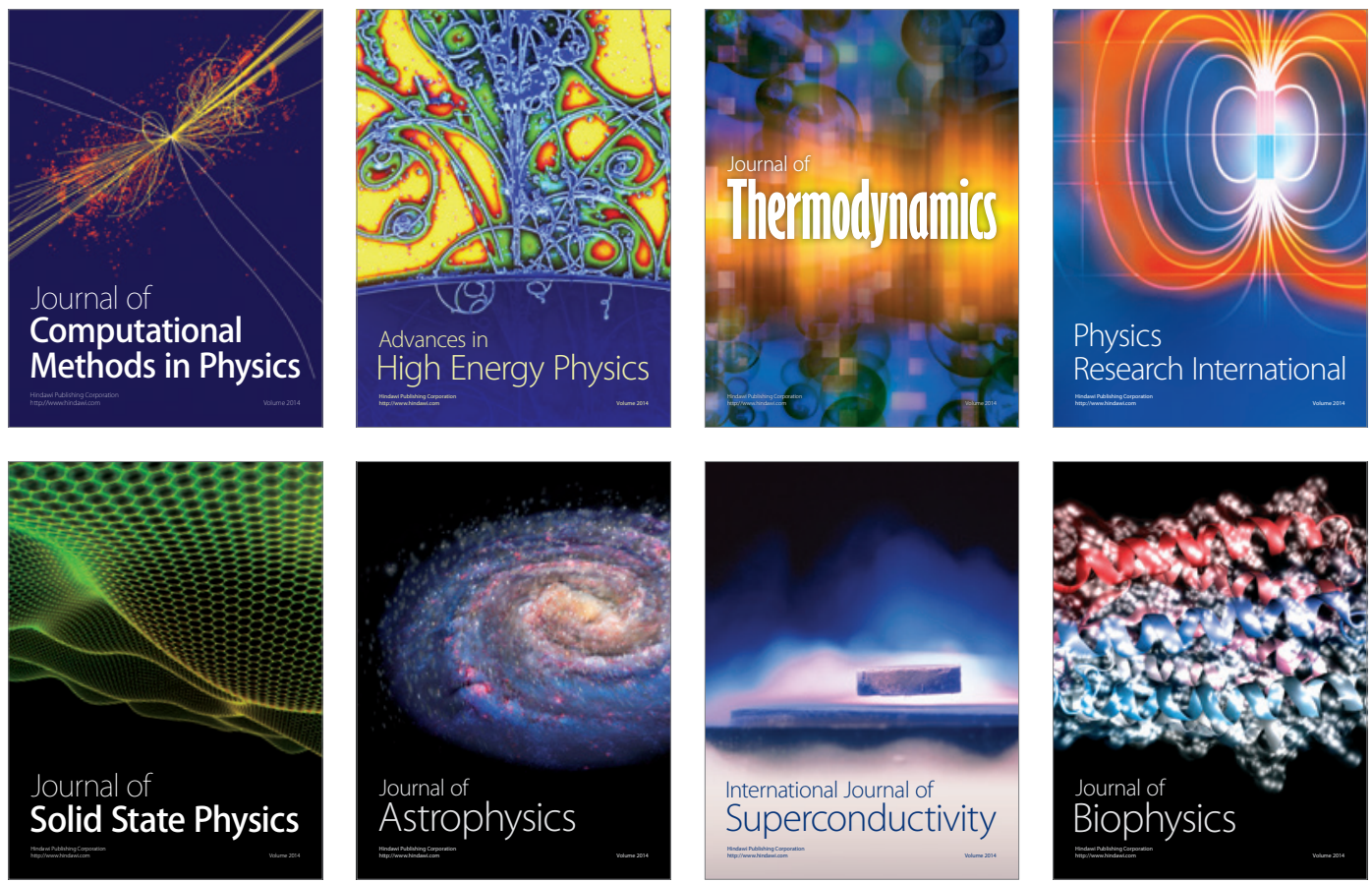
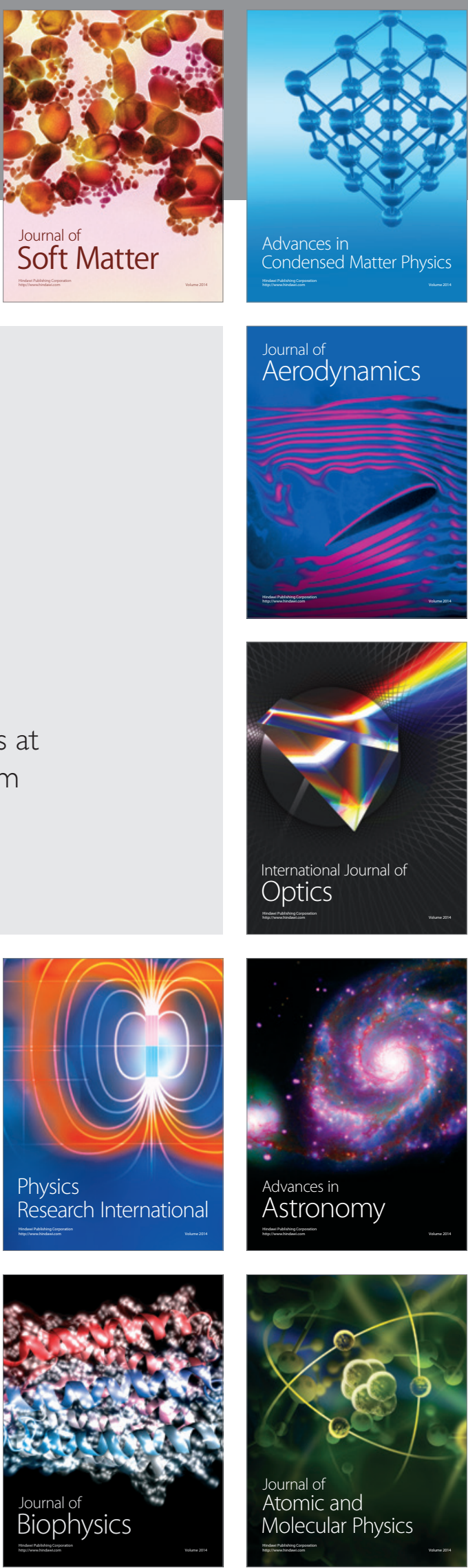